\title{
RESTORATION OF SENSIBILITY IN THE HAND BY NEUROVASCULAR SKIN ISLAND TRANSFER*.
}

\author{
R. Tubiana and J. Duparc, Paris, France \\ From the Clinique Orthopédique et Réparatrice de l'Hôpital Cochint, \\ and the Centre de Traumatologie de l'Hôpital Saint-Louisł
}

The recent development of peripheral nerve surgery owes much to the work of Seddon; in the hand, Moberg (1958) has pointed out the importance of tactile gnosis.

Experience has shown that prehension is useful only in the presence of cutaneous sensibility; it is therefore necessary to re-establish sensibility of the hand whenever possible. If it is impossible to undertake direct repair of the nerves, another procedure must be considered.

Since 1958 we have used in ten patients a sensitive " hetero-digital " cutaneous transplant with its neurovascular pedicle. This procedure was suggested by Moberg (1955) and has been carried out by Littler (1960) and probably by other surgeons.

\section{TECHNIQUE}

The cutaneous transplant-The digital skin island transfer used corresponds to the terminal zone of distribution of the palmar collateral neurovascular pedicle. It is cut oval in shape, takes

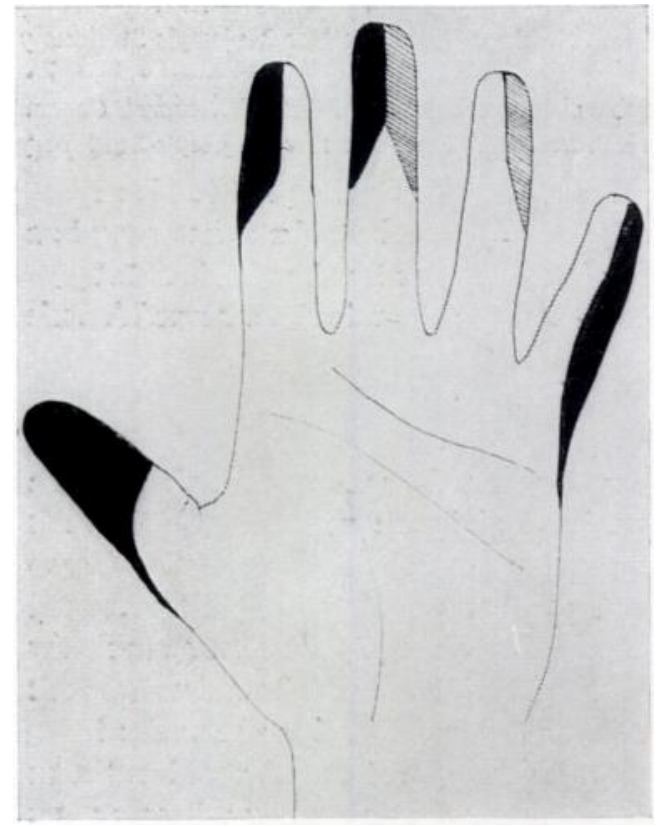

FIG. 1

The relative value of sensibility in various sites. In black, the areas where sensibility is essential cross-hatched, the areas where loss of sensibility is least inconvenient. in half of the pulp and extends upwards onto the middle segment of the finger if necessary.

The donor finger-There must be two conditions present for the choice of the donor finger. The flap must have perfect innervation and blood supply, and removal of the island must give rise to minimal disturbance.

In fact, sensibility does not have the same value in all the fingers (Fig. 1). Sensibility is essential on the palmar aspect of the thumb, on the lateral aspect of the fingers opposing the thumb, and on the medial aspect of the little finger.

On the other hand, loss of sensibility usually gives less disturbance on the medial aspects of the middle and ring fingers, for which reason they constitute the donor areas that we use most. As an alternative, when these sites.are not available, it is possible to use the lateral aspects of the ring and little fingers or even the medial aspect of the index. The approach-A combined digital and palmar incision exposes the entire pedicle. This incision is midlateral in the finger and curved in the palm (Fig. 2).

Liberation of the pedicle requires division between two ligatures of the collateral artery of the neighbouring finger, and cleavage proximally of the digital nerve in the palm (Fig. 3).

* Paper read at the Mecting of the British Orthopacdic Association in Glasgow on April 7, 1960.

† Service of Professor Merle d'Aubigné.

$\ddagger$ Service of Professor J. Cauchoix. 


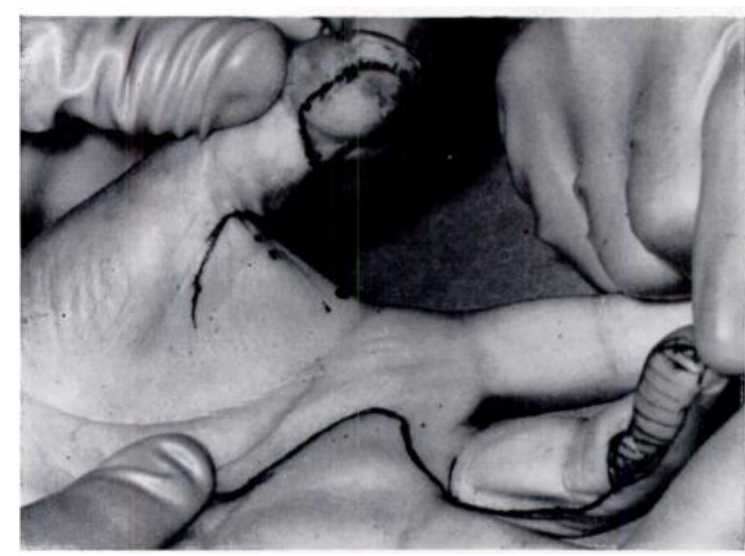

FIG. 2

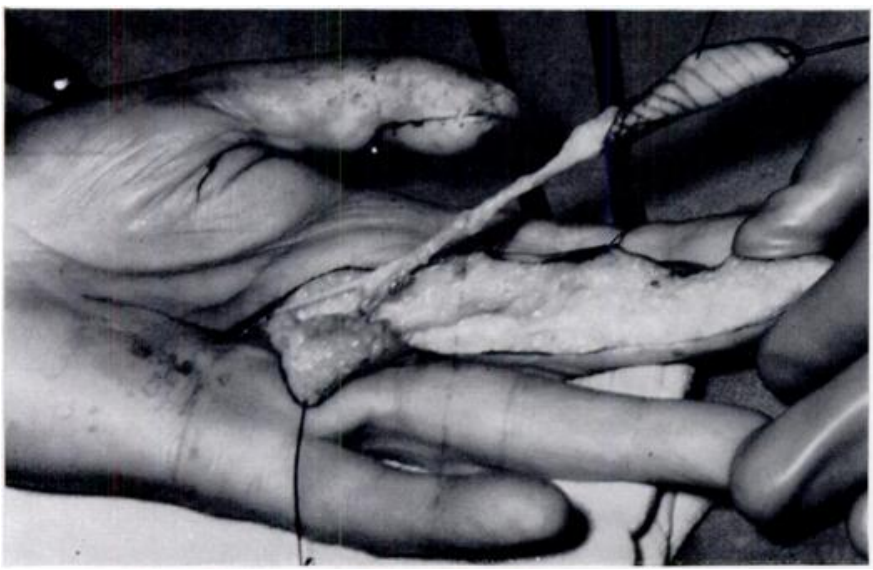

FIG. 4

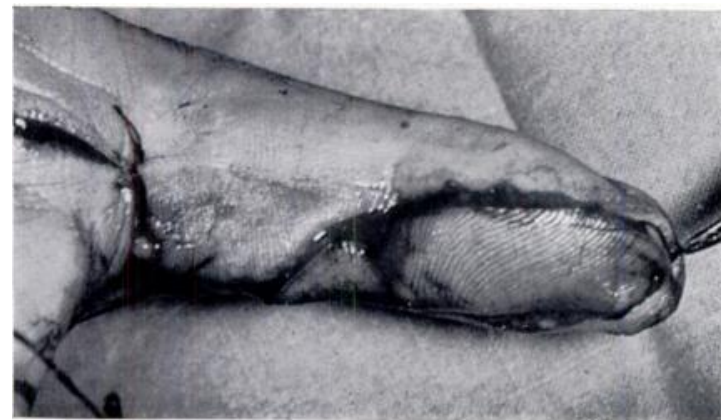

FIG. 6

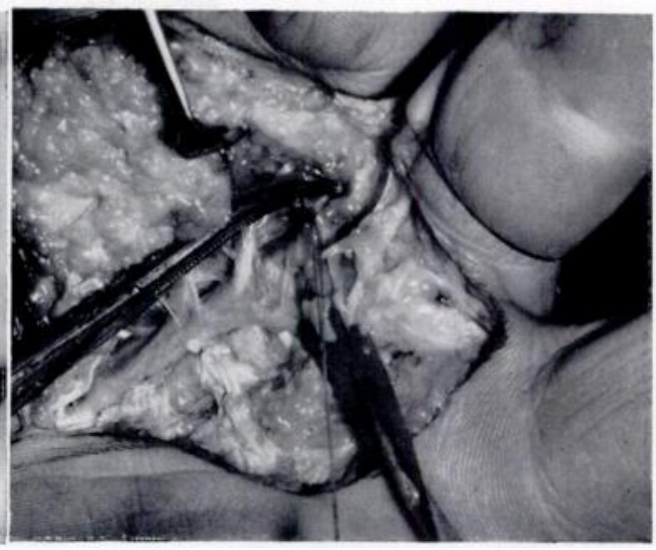

FIG. 3

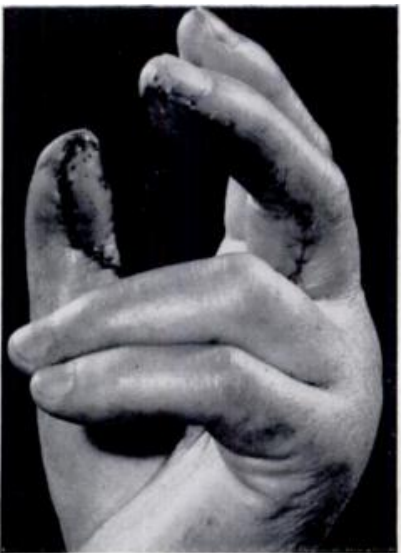

FiG. 5

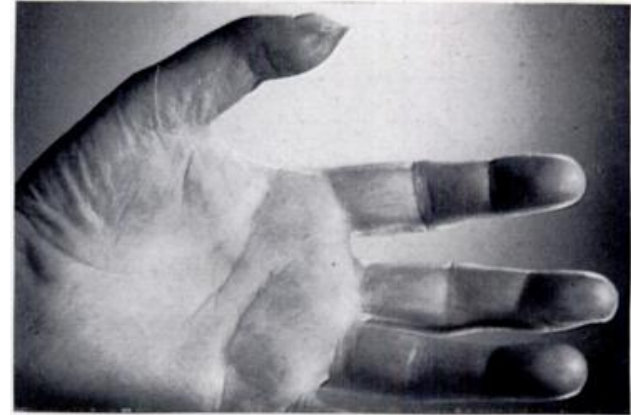

FiG. 7

Figs. 2 To 7

Transfer of sensitive skin to pulp of a thumb rendered insensitive by damage eight years previously to the digital nerves at the base of the thumb. Figure 2-Showing the incision for island transfer from the medial side of the middle finger to the thumb. Figure 3-Ligature and division of the artery to the ring finger. Figure 4-The cutaneous transplant with its pedicle completely freed. Figure 5-The skin of the pulp of the thumb is used as a graft to cover the donor area on the long finger. Figure 6-The transplant is placed at the level of the pulp of the thumb. Figure 7-The appearance of the hand three months after the operation. The whole of the thumb has recovered sensation. 
The dissection of the pedicle is carried to the base of the island which is taken with all its subcutaneous tissue.

While the pedicle is being freed (Fig. 4) it is important to take particular care of the small vessels which arise from it; they are carefully ligated. An anomaly of the arterial distribution may give rise to difficulties. In one patient the digital artery to the fourth cleft arose from the deep, not the superficial arch, and resulted in shortening of the pedicle. This abnormality did not make the transfer of the flap impossible; the difficulty was overcome by flexing both fingers during the post-operative period. Full extension was regained within two months.

After this dissection the tourniquet is released to prove the viability of the island.

Transfer of the island-An area of skin of the same dimensions as the cutaneous island transfer is excised from the finger where the sensibility is to be restored. This excised skin may be used to cover the donor site (Fig. 5). The sensitive cutaneous transplant can be brought through a continuous incision or by tunnelling to the site where it will be implanted (Figs. 6 and 7). It is important to avoid any damage to the pedicle by torsion, excessive tension or compression. A dressing immobilises the fingers for a few days in the most favourable position for the survival of the transplant.

\section{RESULTS}

We shall discuss the restoration of sensibility and of function.

Sensibility-The expected results have been obtained in the ten patients in whom we have practised this operation: the hetero-digital cutaneous transplant has conserved its viability and sensibility, but we have also noticed alterations in sensibility which are of interest.

In the cutaneous transplant-In all the patients, sensibility in the skin island transfer is comparable in quality to the normal. Two-point discrimination is at first a little disturbed, but later becomes almost normal.

In six of ten patients the sensibility perceived by the patients has evolved in a functional and progressive adaptation.

Immediately after the operation the patient appreciates stimuli as if coming from the donor site. After a variable length of time, usually a few weeks, the sensibility of the receiving finger is added to the sensibility of the donor finger. The perception of this "double sensibility" modifies itself progressively and the patient becomes more and more aware of his receiving finger. In agreement with Moberg, it seems useful to distinguish between academic recovery and functional recovery after any operation for the restoration of sensibility. If, perhaps, the sensation between the giving and receiving fingers is confused during the neurological examination, mistakes of perception are not usually made from the functional point of view. However, the passing from one stage to another in the evolution is not constant, and delays, which are very variable, seem to be influenced by the patient's psychological condition and the restoration of the use of the finger.

In four cases, even after many months, the sensibility of the receiving finger is still felt as of the donor finger. It is interesting to note that in all these four patients the finger operated upon was, for various reasons, rarely used.

Around the transplant-We have noticed the presence of a progressive sensibility which spreads from the cutaneous transplant at varying rates. It is noticeable that this new sensibility is usually immediately localised to the receiving finger. The quality of this sensibility is not as good as in the island, but it is protective.

Many explanations can be offered for this spread of sensibility. It seems that it comes from the transplanted nerve; the abundant terminal branching of the digital nerve may explain this high potential of diffusion. The penetration into the neighbouring tissues is more rapid when it is not limited by sclerosis, and probably explains the individual differences; but we have often noticed that the perception in the cutaneous transplant and perception 


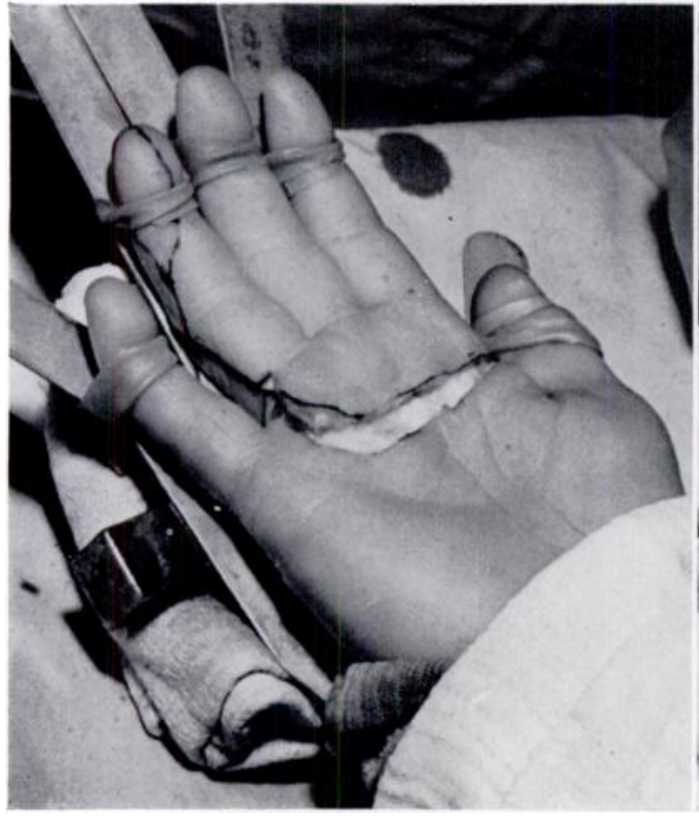

FIG, 8

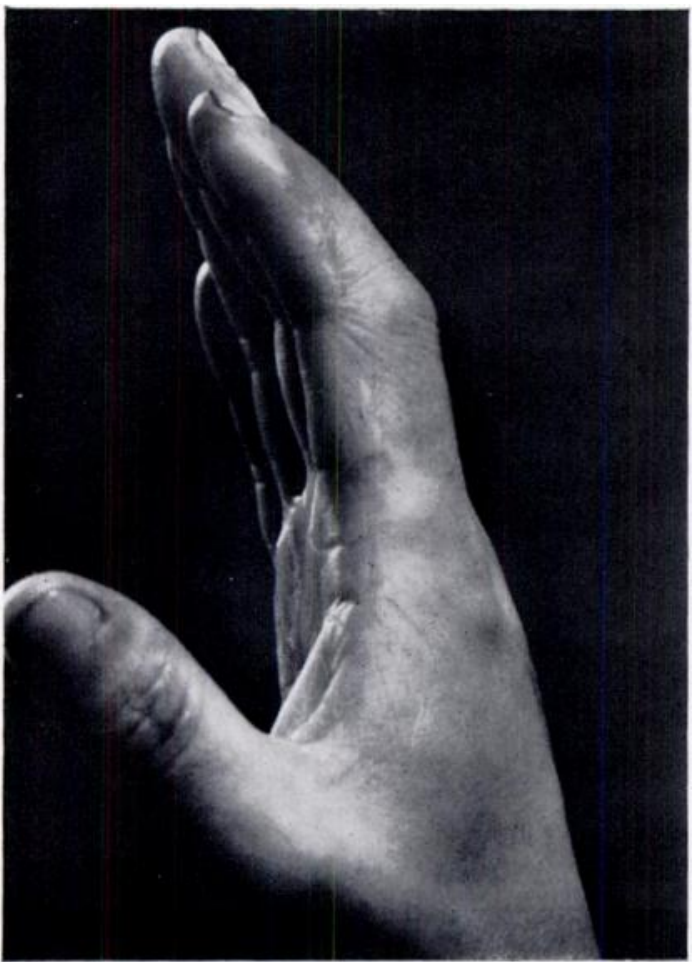

Fig. 10

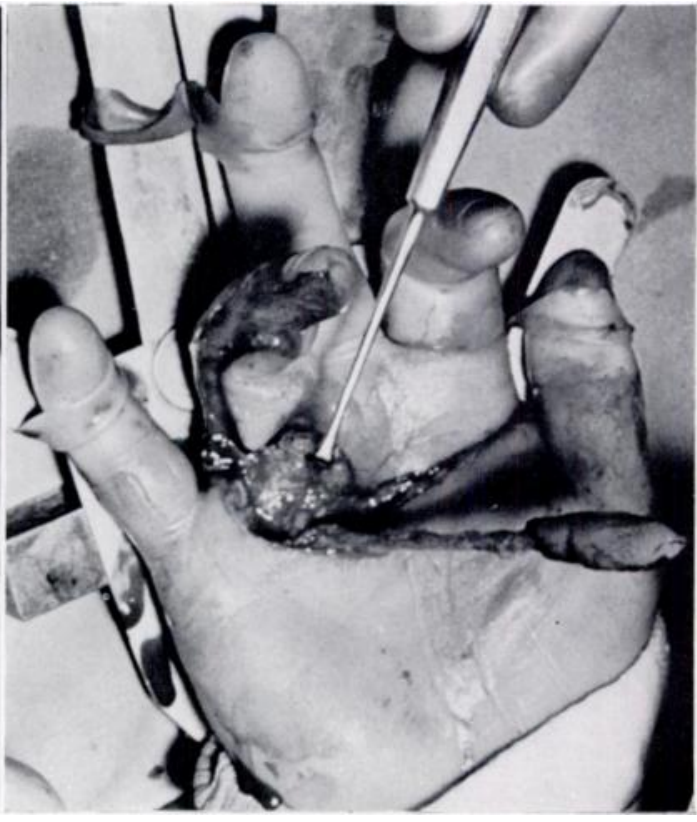

FIG. 9
Figs. 8 To 10

Section of the median nerve at the wrist eight years earlier. Transfer of a cutaneous transplant taken from the medial side of the ring finger and inserted on the lateral side of the index. Figure 8-The incision. Figure 9-The cutaneous transplant with its pedicle entirely freed. Figure 10-The appearance of the hand four months after operation The whole index finger has recovered sensation. 


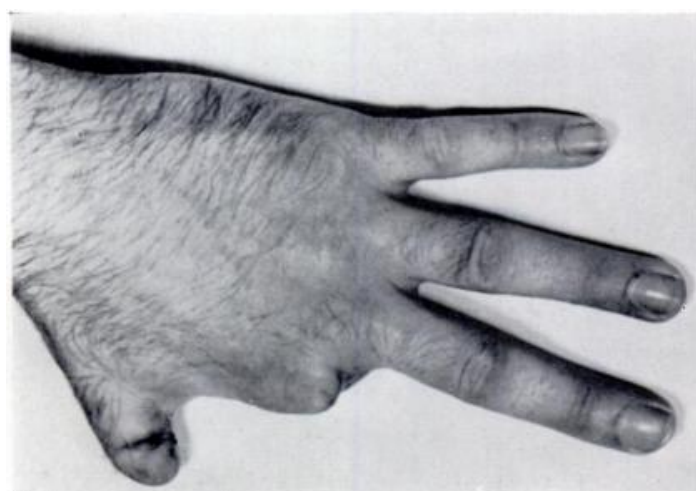

FIG. 11

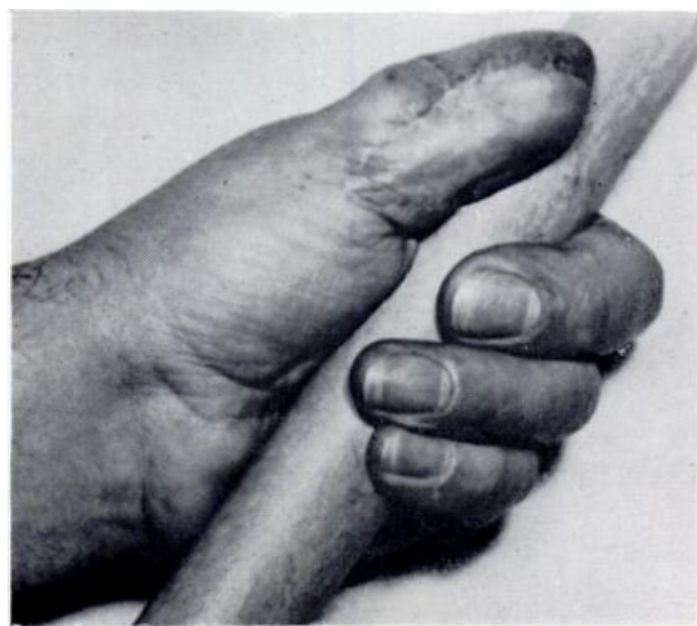

FIG. 13

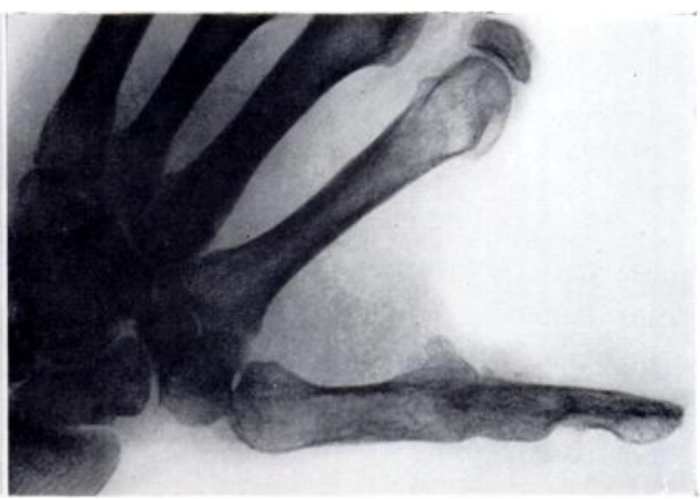

FIG. 12

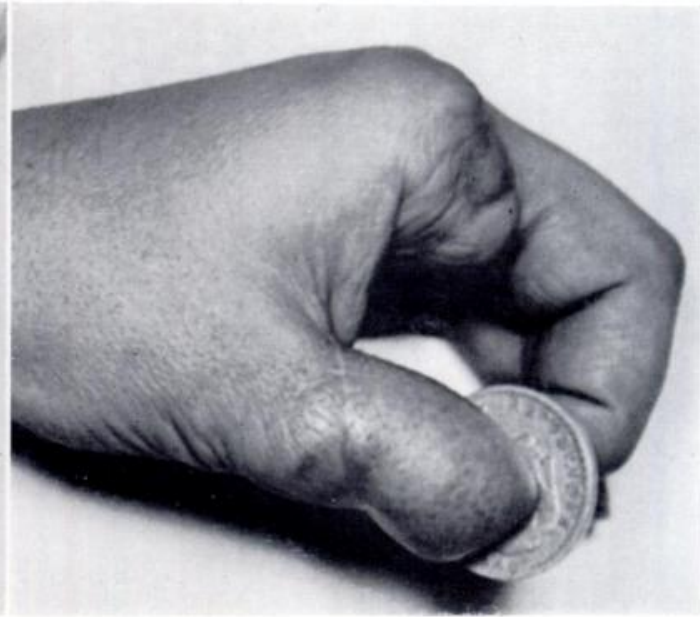

FIG. 14

Reconstruction of thumb after traumatic amputation of index finger and thumb. Figure 11-Condition before operation. Figure 12-Reconstruction of the thumb by a tube pedicle raised from the opposite forearm, insertion of a bone graft from the ilium and skin island transfer from the medial side of the ring finger. Figures 13 and 14-Six months after operation, all the tissues of the reconstructed thumb have recovered sensibility, including those of the tubes. Pinch and grasp are possible.

around it are different, since the peripheral sensibility is at first, in most cases, that of the receiving finger; it is impossible at present to explain this difference in localisation.

These discussions which interest neurologists to whom we have shown these patients must not allow us to forget the functional side of this operation.

The functional results-These depend on several considerations.

The quality of perception-Function is really good only when the patient is adapted to this new perception. This adaptation takes, as we have seen, a variable time. The extension of sensibility around the flap improves the functional result considerably.

The nutrition-The transfer of such a neurovascular cutaneous transplant improves considerably the nutrition of the injured finger. This procedure gives not only sensibility but also an important vascular supply. We have noted in every case a marked increase in the suppleness of the tissues and sometimes of the joints of the recipient finger.

Pain-In two patients with anaesthetic and painful hands we had hoped that re-establishing the sensibility of the finger would improve the function and diminish the pain. This goal has not been achieved, and not only has causalgia persisted, impairing function, but the transferred skin and donor area also became painful. Consequently it seems necessary to treat the pain of anaesthetic and painful hands before considering the restoration of sensibility. 


\section{INDICATIONS}

We have used this operation for two reasons: the treatment of insensitive digits and reconstruction of the thumb. Details of our ten cases will be published in another article. Treatment of insensitive fingers-Anaesthesia of the fingers may result from paralysis of the median or ulnar nerves or from destruction of digital nerves.

Paralysis of the median nerve - In a patient eight years after section of the median nerve at the wrist, a neurovascular skin island was taken from the medial side of the ring finger and transplanted to the index finger; the entire index finger regained sensibility. In such a patient it would be more logical to transfer the sensitive transplant to the thumb, giving a sensitive grip between the thumb and all the opposite fingers.

Paralysis of the ulnar nerve-A sensitive cutaneous transplant can be taken from the medial side of the middle finger and transferred to the medial side of the little finger. This procedure has been performed in two patients. In the first the island was transferred to the little finger which was stiff and retracted. The sensibility of the transplanted skin has a protective effect in spite of the fact that it is still felt as of the third finger and that there is almost no extension of sensibility around the island; but this retracted finger is rarely used.

In another similar case we preferred to amputate an insensitive and stiff little finger and at the same time transferred from the middle finger a sensitive cutaneous transplant to the medial side of the ring finger, while the sublimis tendon of the little finger was transplanted onto the dorsal expansion of the ring finger. In a few weeks the entire ring finger regained sensibility which was mostly felt as arising from the ring finger.

Destruction of digital nerves-We operated on four patients, the thumb in three and the index in the fourth.

A sensitive cutaneous transplant can be taken from the medial palmar aspect of the middle or ring finger and transferred to the thumb or another finger which has had both digital nerves destroyed. These patients have less good functional results, probably because of scar tissue in the fingers.

In addition to the two patients mentioned who had painful hands before operation and remained so after (one has since improved after a thoracic sympathectomy), a third patient developed pain at the level of the passage of the pedicle under the palmar incision at the base of the thumb; a secondary operation has freed the pedicle and a rotation cutaneous flap has avoided further irritation.

One patient with an anaesthetic and painful thumb (Fig. 2) had the two neuromata of the collateral nerves resected and the proximal part of each nerve transferred through the thenar muscles to the dorsal aspect of the hand with good results. Then a neurovascular skin island transfer brought excellent restoration of sensibility and function.

Reconstruction of a sensitive thumb - In three cases we have combined osteoplastic reconstruction of a thumb with the transfer of a hetero-digital cutaneous transplant; this technique requires several operations.

Preparation of a tube pedicle flap-It is necessary to fashion a flap of a suitable length. The most suitable donor area is the infra-clavicular region, but the tube can be raised from the abdomen or the upper limb. If the tube is not too long it is possible to make a direct implant on the thenar eminence without incurring excessive risk, but it is safer to do this in two stages. During this transfer it is important that the longitudinal suture line of the tube is on the palmar surface, because it is at this site that the island transfer will be placed.

Separation of the tube is done three weeks later.

Inclusion of the bone graft and of the skin island transfer-This is performed after complete healing of the tube. It consists of three stages as follows: 1) Opening of the tube. The tube is opened along the line of the suture exposing the end of the bone into which a bone graft will be placed. 2) Inclusion of the bone graft. The graft is usually taken from the iliac crest. It is fixed by pushing the tip of the tapered graft inside the medullary canal of the first

VOL. 43 B, NO. 3, AUGUST 1961 
metacarpal. The length of the graft and of the reconstructed thumb depends on the site of amputation; the aim is to provide a thumb sufficiently long to allow easy opposition. Because it has no joint, the thumb must be a little shorter than normal. Other procedures often result in either a thumb which is too short or a thumb which is too long for the available blood supply and suffers trophic changes. 3) Transfer of the island flap. The procedure is the same as described for the other fingers except that it should be larger-four to five centimetres long and about one centimetre broad. The neurovascular skin transfer is introduced into the tube along the line of previous suture allowing easy closure after the bone graft has been sited.

The reconstructed thumb is immobilised in plaster-of-Paris for about one month. In all cases union has been rapid.

The result in these three patients has been equally satisfactory in prehension and sensibility. The nutrition also has become excellent. The transfer of a good blood supply is probably the explanation of the rapid consolidation of the bone graft and the lack of trophic changes. Any osteoplastic reconstruction of the thumb already performed can be benefited by the transfer of such a neurovascular island. In spite of its relative complexity this new procedure of thumb reconstruction seems to have useful applications.

\section{SUMMARY AND CONCLUSIONS}

In ten cases definitive loss of sensibility in an important territory of the hand has been treated by a hetero-digital, neurovascular skin island transfer. Operative technique and results obtained are reported. Although not enough patients have been treated to allow us to give precise indications for the procedure, two such indications clearly emerge: 1) the treatment of insensitive digits; 2) the reconstruction of the thumb or fingers. The procedure could probably be used elsewhere than in the hand.

\section{REFERENCES}

LitTLER, W. (1960): Neurovascular Skin Island Transfer in Reconstructive Hand Surgery. In Transactions of the International Society of Plastic Surgeons. Second Congress, London 1959, p. 175. Edited by A. B. Wallace. Edinburgh and London: E. \& S. Livingstone Ltd.

Moberg, E. (1955): Transfer of Sensation. Journal of Bone and Joint Surgery, 37-A, 305.

Moberg, E. (1958): Objective Methods for Determining the Functional Value of Sensibility in the Hand. Journal of Bone and Joint Surgery, 40-B, 454.

Tubiana, R., and Duparc, J. (1959): Opération palliative pour paralysie sensitive à la main. (Rapport de R. Merle d'Aubigné.) Mémoires de l'Académie de Chirurgie, 85, 666.

Tubiana, R., and Duparc, J. (1960): Un procédé nouveau de reconstruction du pouce. Annales de Chirurgie Plastique, 5, 287. 